\title{
Ethanol ingestion as an operant response'
}

\author{
R. J. SENTER, F. W. SMITH ${ }^{2}$ AND STANLEY LEWIN ${ }^{3}$ \\ UNIVERSITY OF CINCINNATI
}

Data are reported for laboratory rats required to ingest a 7\% EtOH solution to avoid shock, attain food, or for no primary reinforcement. When alcohol ingestion was coupled with shock avoidance or no reinforcer, no increase in ad lib alcohol consumption resulted. The use of ethanol ingestion as a food producing operant, however, yielded a significant but temporary increase in voluntary EtOH consumption subsequent to the conditioning procedure. The use of a positive reinforcing agent as an inducement toward voluntary EtOH consumption in the rat is suggested as possibly being superior to the more traditional stress avoidance techniques.

Numerous experiments in which animals have been exposed to "stressful" environs while having access to ethanol have been reported. Such experiments are exemplified by the classic Masserman \& Yum study (1946) using cats; Lester (1961) and Dember \& Kristofferson (1955) using rats; and Mello \& Mendleson (1966) using monkeys. The intent involved in this type of experimentation is usually either to determine whether animals will resort to the anesthetic properties of alcohol as an "escape," or to "addict" the animals through the association of alcohol ingestion with the reinforcement resulting from stress avoidance. Experiments purporting to associate stress avoidance reinforcement with alcohol ingestion have often left the temporal relationship between operant emission and occurrence of reinforcement to chance. When the anesthetic properties of EtOH are relied upon to provide the sole avenue for stress avoidance, the temporal distance between the operant (drinking) and the reinforcement (anesthesia) may well be long and variable due to the intervening metabolic processes. Under such circumstances, it would be difficult for a rat to associate operant and reinforcer with sufficient strength to form a persistent habit.

The present study is designed so that reinforcing circumstances are consistently coupled with the alcohol consummatory response. The study was an attempt to establish EtOH consumption as an autonomous habit through operant conditioning.

\section{Method}

Forty-eight male hooded rats ranging from 90 to 120 days of age at the beginning of the experiment served as Ss. All Ss were allowed ad lib access to food, water, and 6\% (by volume) EtOH solution in housing cages for 16 days prior to experimental manipulation. After this pre-experimental ad lib period, the animals were separated into three groups designated SA (shock-alcohol), FA (food-alcohol), and ZA (zero-alcohol). Animals in the SA and FA groups were allowed to drink water in commercial Skinner boxes (Scientific Prototype). A mild shock was then administered through the grid floor to the SA animals and the drinking fount was filled with a $7 \%$ EtOH solution. The fount was connected to an electronic apparatus (Senter, Munz, \& Eimer, 1966) such that continuous drinking (1.e., one contact with fluid per each half sec) would maintain an $8 \mathrm{sec}$ "shock-off"' condition. Thus the $\mathrm{S}$ was required to drink for at least $1 / 2 \mathrm{sec}$ every $8 \mathrm{sec}$ to maintain a constant "shock-off" condition. Continuous contact (e.g., paw in fluid) or no contact would not maintain the "shockoff" condition. The shock was increased daily until a level of $50 \mathrm{~V}$ was reached. With this arrangement the animal could achieve continuous "shock-off" only by sustained drinking.

The FA animals were trained such that sustained drinking would produce a $45 \mathrm{mg}$ food pellet (Senter, Munz, \& Eimer, 1966). The duration of continuous drinking required for the delivery of a single pellet was increased daily to a maximum of $8 \mathrm{sec}$.

Subsequent to the initial shaping, the SA and FA groups were exposed to their respective conditioning circumstances for $1 / 2$ to $1 \mathrm{~h}$ every day for 60 days.

The animals in the ZA group were each "yoked" to a member of the SA group. Every day each rat in the ZA group received, while under $23 \mathrm{~h}$ of thirst, a ration of $7 \%$ alcohol solution equivalent in volume to the amount consumed by its SA pair-mate in the Skinner box. The alcohol ingestion pattern of the ZA animals was, then, identical to that of the SA animals for the 60 day experimental period. All animals were allowed ad lib access to food and water for $1 / 2 \mathrm{~h}$ after each day's running.

After the 60 days of experimental manipulation all animals were returned to their home cages and again given ad lib access to food, water, and $6 \%$ EtoH for 16 days.

A control group receiving a sucrose solution instead of EtOH was also run under each of the conditions described above. These animals showed such a high preference for the sucrose throughout the experiment that it would have been impossible to detect any increases which might have resulted from the experimental manipulations. The data from these control groups were omitted from the analyses.

\section{Resulfs}

All SA and FA Ss learned, and performed reliably, their operants in the Skinner boxes. The quantities of EtOH solution consumed by all groups during the 60 day experimental period were frequently sufficient to produce overt symptoms of intoxication.

The data of primary interest were collected before 


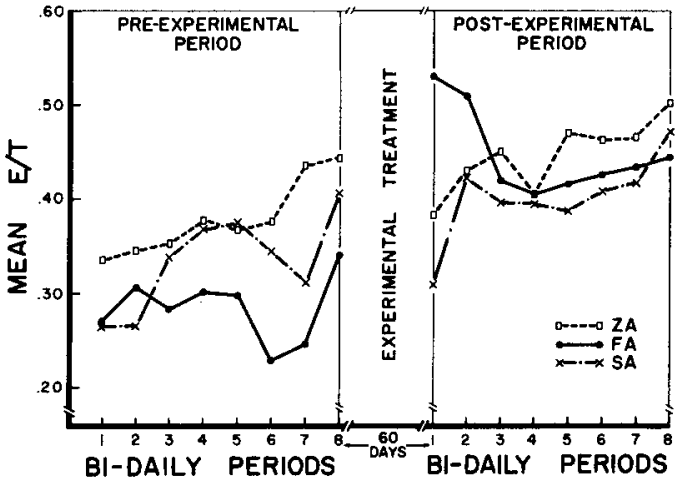

Fig. 1. Ratios of ethanol to total fluid intake for all animals during the pre- and post-experimental periods of ad lib access to $6 \%$ EtOH and water.

(pre-ad lib) and after (post-ad lib) the experimental manipulations. An index of preference for the EtOH was expressed as $E / T$, or the daily intake of ethanol divided by the total (EtOH and water) fluid intake for each day.

As can be seen from Fig. 1, none of the experimental treatments was effective in producing persistent alcohol preference. The $\mathrm{SA}$ and $\mathrm{ZA}$ groups show, if anything, a slight decrement in the $E / T$ ratio immediately subsequent to their experimental manipulations.

The data were subjected to a square root transformation and then to a 3 (treatments) by 2 (pre-post) by 8 (time in bi-daily periods) analysis of variance. The pre-post comparison of $E / T$ resulted in $F=5.624$, $(d f=1 / 45, p<.05)$. The other two main effects (treatments and time) failed to produce significant $(\alpha=.05)$ F-ratios. The interaction of these two variables (treatments by time), however, resulted in $F=1.842$ (df $=$ $14 / 315, \mathrm{p}<.05)$.

Inspection of Fig. 1 suggests a likely source for this interaction. It lies in the data points for the FA group during the first few days of the post-experimental period. The FA group shows an initial increase in $\mathrm{E} / \mathrm{T}$ followed by a decline. $\mathrm{SA}$ and $\mathrm{ZA}$ animals show an opposite tendency. The data for the first two post-ad lib days for all three groups were subjected to a 1 by 3 analysis of variance yielding $F$ $=4.40(\mathrm{df}=2 / 45, \mathrm{p}<.05)$. Duncan's multiple range test indicated, further, that the FA E/T for the first two post-ad lib days differed significantly from both $\mathrm{SA}$ and $\mathrm{ZA}$ ( $p<.01$ and $p<.05$, respectively), but that SA did not differ significantly $(\alpha=.05)$ from ZA. The changes in $\mathrm{E} / \mathrm{T}$ between the last two days of pre-ad lib and the first two days of post-ad lib, for each group, were examined via Wilcoxon $T$ and Sandler's A. This change, for the FA group, yielded $T=32$ and $A=.32$ $(p<.03$, one tailed in both cases). The other two comparisons did not produce significant differences $(\alpha=.05)$.

\section{Discussion}

The experimental manipulations applied to the SA group in this study represent a rather strict application of the conditions suggested by the stress avoidance theory as contributing to the etiology of alcoholism. The animals were subjected to a presumably stressful situation (shock) which could only be escaped by ingesting an EtOH solution. This treatment produced no indication whatever that the prolonged use of alcohol ingestion as an escape operant enhanced the animals' subsequent ingestion of the substance. It is conceded that the "stress" used in this study was, for each day, acute rather than chronic. It is possible that continuous prolonged exposure (days or weeks) to such stress, with escape available only through ethanol ingestion, might produce different results.

In this study the only hint of facilitation of the alcohol ingestion response was produced by associating that response with positive reinforcement (viz., food). This "enhancement," if it exists at all, is temporary and appears to undergo the characteristic course of extinction once the reinforcer is removed.

The results of this study, coupled with those from other reports (e.g., Lester, 1961; Senter \& Sinclair, 1966), and research currently ongoing in the writer's laboratory, lead to the suggestion that greater fruition might be achieved in the striving to develop an "alcoholic syndrome" in non-human mammals if alcohol ingestion is used in association with positive reinforcement rather than in the more traditional "escapefrom-stress" paradigm. Should future research confirm the observation that positive reinforcement enhances the "habit" of alcohol consumption more than does "escape-from-stress," an alteration of some of the very basic attitudes concerning the nature of the variables contributing to the etiology of chronic alcoholism may well ensue.

\section{References}

Dember, W. N., \& Kristofferson, A. B. The relation between freechoice alcohol consumption and susceptability to audiogenic seizures, Quart. J. Stud. Alcohol, 1955, 16, 86-95.

Lester, D. Self-maintenance of intoxication by rats. Nature, 1957, $180,1292-1293$.

Masserman, J. H., \& Yum, K. S. An analysis of the influence of alcohol on experimental neuroses in cats. Psychosom. Med., $1946,8,36-52$.

Mello, Nancy K., \& Mendelson,' J. H. Factors affecting alcohol consumption in primates, Psychosom. Med., 1966, 28, 529-550.

Senter, R. J., Munz, D., \& Eimer, E. Stimulus control through the licking response. Psychon. Sci., 1966, 6, 489-490.

Senter, R. J., \& Sinclair, J. D. Self-maintenance of intoxication in the rat: A modified replication. Submitted to Quart. J. Stud. Alcohol, April 1967.

\section{Notes}

1. This research is a portion of the effort carried out under $\mathrm{MH}$ 11063-01.

2. Now with Electronics Division AVCO Corp.

3. Now at Miami University of Ohio. 